\title{
Voice based E-mail for the Visually Impaired
}

\author{
Aishwarya Belekar, Shivani Sunka, Neha Bhawar, Sudhir Bagade \\ Department of Computer Science \& Technology, \\ Usha Mittal Institute of Technology, \\ SNDT Women's University, Mumbai, India
}

\begin{abstract}
The increased use of technology and it's limitless opportunities have made it inevitable for the present generations to apply the Internet technology to the fullest. Email being one of the most widely used features of the Internet serves as the basic prerequisite. Apart from normal users, the visually impaired one's face challenges when it comes to using the internet in spite of the availability of various screen readers. This paper thus aims to provide voice assistance for them. Along with Email, voice assistance for various simple yet important applications used on a daily basis such as Calculator, Music etc.
\end{abstract}

\section{Keywords}

Voice based Email, Visually impaired, Speech-to-text, text-tospeech, Speech recognition.

\section{INTRODUCTION}

The most common mail services that are used in our day to day life cannot be used by visually challenged people. To make these systems convenient for these people who are visually challenged there are various technologies provided to them like screen reader, automatic speech recognizer, speech to text and text to speech, braille keyboard, etc. However, these technologies are not that much useful for those people as it could not give the proper response like a normal system.[1]

The objective of Voice Based Email for Visually Impaired is to help challenge one's access mails easily and efficiently.

This application is based on using speech-to-text and text-tospeech converters, thus enabling everyone to control their mail accounts using their voice only and be able to read, send, and perform all the other useful tasks. The system will prompt the user with voice commands to perform certain action and the user will respond to the same.

So here put to use are the Speech-to-Text and Text-to-Speech technologies using .net framework.

The Speech-to-Text also known as Automatic Speech Recognition converts spoken speech into text, which helps compose emails as an easy task.

The Text-to-Speech module gives audio output of the mail received, the sender, the subject and the body of the mail is read out by the system.

Along with it, the aim is to provide assistance to use fundamental applications like My Computer, Word, Notepad, etc.

The rest of the paper is organized as follows. Section 2 presents the literature survey. In section 3, we proposed our system for the visually impaired. Implementation details are presented in section 4 , and Conclusion is in section 5 .

\section{LITERATURE SURVEY}

In this section, we present the detailed literature survey of the present similar techniques.

In paper [2], a voice based email architecture is proposed which will help blind people to access email. The existing system is not user friendly for blind people as it does not give any audio feedback to readout contents for them. The proposed system makes use of Speech Recognition, Interactive Voice Response and Mouse Click events. Also, for additional security purposes voice recognition is used for user verification. In this system, Registration is the first module. This module will collect complete information of the user by prompting the user to what details need to be entered. The second module is the login module in which the system will ask the user to provide user name and password. This is done through voice commands.

Another voice sample is asked for performing the voice verification. Then the user is redirected to the inbox page once login is done. After login, users can perform normal operations of a mailing system. System options are: Compose, Inbox, Sent Mail, Trash. The user can switch between these using voice commands.

In paper [3], proposed the system that relies on a voice command based system unlike the existing mail system. The complete system is primarily based on speech to text commands. Once using this system the application will be prompting the user to speak specific commands to avail respective services and if the user wants to access the respective services the user needs to speak that command. This application makes use of IMAP (Internet Message Access Protocol).It is an Internet standard protocol used by email clients to retrieve clients to retrieve email messages from a mail server over a TCP/IP connection. The Main activity Screen will be the First screen to be displayed on start of the app. This screen waits for the user to press the button so that the system will start accepting voice commands. And this is a full sized button so they can press anywhere on the screen. Then using Voice commands users can send, read emails.

In paper [4], the system uses mainly three technologies:

- Speech to text

- Text to Speech.

- Interactive Voice Response.

When the user visits the site for the first time he/she would need to register through voice commands. Also after registration, the user's voice will also be recorded and stored in the database. And the user will get an Id and password. After login, the user can access the mail option. In this system. The user interface is designed using Adobe Dreamweaver CS3. The complete website mainly focuses on efficiency in understanding. Also there is a contact us page where the user 
can suggest any suggestion or any help if they need.

In paper [5], have proposed an email system which can be accessed easily by blind people. The use of Speech to Text convertor, Text to speech convertor and Viterbi Algorithm are taken into consideration.. The algorithmic rule works with the technique that the system detects the foremost acceptable word once the user spells it so matches the word that's guessed with the particular word that's pronounced. The user needs to register to the website when they visit the site for the first time. This system reduces some drawbacks of the existing system. The drawback of this system is that the efficiency of the Viterbi algorithm decreases as the number of errors increases as well as it requires more space [5].

Saurabh Sawant et.al in paper [6] proposes a system for visually impaired and illiterate people for improving their interaction with the email system. This system eliminates use of IVR Technology that used Screen Readers and Braille Keyboard. There, have used Speech to text and Text to speech conversion. Also for other operations voice commands. For registration, used email id and password. For the functionality, use a feature of PHP that is PHP mailer. It is a library which can be used to send email. In order to fetch the user's mail from the IMAP server. Here, Knuth-Morris-Pratt Algorithm is used for searching mail in inboxes. To conclude, the system environment is purely voice based driven with proper feedback from the system at every stage. The drawback of this system is that it uses Gmail as a host server so we cannot use other email services like Yahoo etc. [6].

Payal Dudhbale et.al. [6] Proposed Voice Based System in Desktop and Mobile Devices for Blind People. The major components of the proposed system in this paper [7] are mentioned below.

1. G-mail System reads messages on recipient mailbox.

2. RSS-Real simple syndication for news

3. Song-listen songs

4. Book reader-system red book

5. Drive browser-To search drives and folders.

The architecture is used by blind people to access Email and multimedia functions of the Operating system easily. The GUI design can be accessed using voice commands and mouse clicks eliminating the use of a keyboard. Along with Email, also developed RSS that's Really Simple Syndication which is a way to distribute a list of headlines, update notices. Also developed a mobile application for the same. Along with Email other applications can also be accessed through voice commands [7].

In [8] paper, the authors have proposed Tetra Mail, a usable blind-friendly email client to overcome the challenges pertaining to the accessibility and usability of email-related activities on a smartphone.

Proposed System: The proposed email client is evaluated through an empirical study of 38 blind participants by performing 14 email activities. The proposed solution may help blind people in sending, receiving, organizing, and managing emails. The usability of the design has been evaluated using standardized HCI usability and accessibility parameters. The proposed solution was compared to other email clients such as Gmail and Thunderbird.

Framework for Tetra Mail client: Based on the HCI model, they have developed a framework for an email client for blind people called Tetra Mail. The interface of Tetra Mail is designed in such a way that a blind user can easily use it without in-depth knowledge of operating touch screen interfaces.

Results: The results reflect that Tetra Mail is a better alternative for blind users due to its consistent and blindfriendly interface design. The results of this prototype implementation show an improved user experience, accuracy in task completion, and better control over touch screen interfaces in performing basic activities of managing emails. The results demonstrate that Tetra Mail is an accessibility-inclusive email client enabling blind people to have a better user interaction experience and minimal cognitive overload in managing emails. The solution is tested through an empirical study. Results showed that this email client helps blind people to send and receive emails with comfort and ease. [8]

\section{Advantages of the above surveyed techniques}

In most of the papers, it can be seen that the whole process of speech-to-text and text-to speech makes it more interactive and easy for the visually impaired people. This system makes the disabled people feel like normal users. Also, voice based is useful for handicapped and illiterate people. Automatic-speech recognizer is of the major advantages. We can see a reduction in cognitive load taken by blind to remember and type characters using a keyboard. Voice based email system is a user-friendly system.

\section{Disadvantages/Limitations of the above surveyed techniques}

In almost all the papers, it can be seen that there is use of mouse clicks for many tasks. It gets difficult for visually impaired people.

Also, the Indian subcontinent is not benefited by this as there are so many languages and speech recognizers cannot recognize these languages. Mostly English language is preferred.

The following section is the proposed system for the visually impaired.

\section{PROPOSED SYSTEM}

In the proposed system, a desktop application is to be developed that can be used by people with various visual imparities, to access emails easily and efficiently. All the existing voice based email systems, provide their own user developed email services and do not incorporate the use of Google's Gmail. So, considering this here, the intention is to develop the application by linking it with the Gmail Client, thereby giving users an additional advantage.

\section{Module Description}

\section{Speech- to-Text Converter}

Speech-to-text converter recognizes the speech, analyzes the sounds you make by filtering what you say, then digitizes it to a format it can read. The recognized text can be saved in a file. .Net and C\#.Net platforms are used here to develop this. Our speech to-text system directly obtains and converts speech to text. Speech recognition systems can be divided into several blocks: feature extraction, acoustic models database which is created based on the training data, dictionary, language model and the speech recognition algorithm.

\section{Text-to-Speech Converter}

Using speech synthesis techniques, it converts text to voice output. It is employed by the blind to concentrate to written material; it's now used extensively to convey financial data, e- 
mail messages, and other information through the telephone for everybody. Text-to-speech is also used on devices such as portable GPS units to announce street names when giving directions.

\section{Voice based Email Application}

The user can send emails, listen to what they have written and also receive emails and listen to them with voice commands. In Email, the application makes use of the SMTP protocol for sending emails and POP3 protocol for receiving emails. SMTP (Simple Mail Transfer Protocol) is the reliable protocol to send emails and it works in a simple way that the SMTP server passes on the email messages quickly. POP3 (Post Office Protocol) is used to receive emails. The POP3 server stores the email and on request the emails are displayed. The same is implemented in our application, that on the request by the user the emails are downloaded.

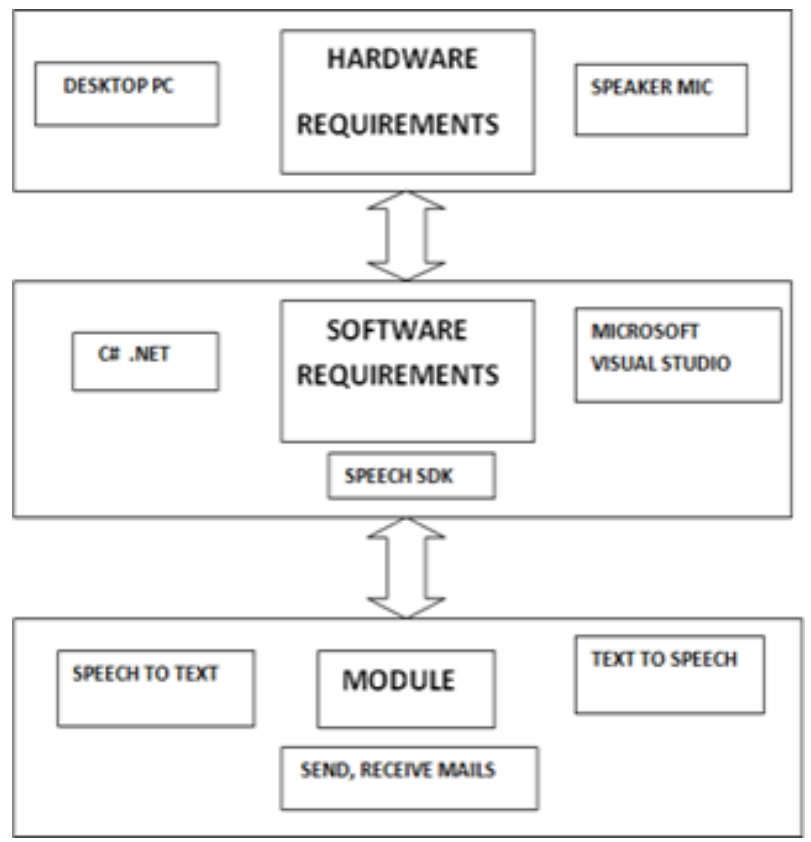

Figure 1: Overview of Proposed System

Fig. 1 represents the overview of the proposed system. Its requirements are detailed as follows:

\section{Hardware Requirements}

- 1 GB RAM

- 200 GB HDD.

- Intel 1.66 GHz Processor Pentium4

\section{Software Requirements}

- Windows10

- Visual Studio2013

- Windows Operating System

- $\quad$ ASP.net

It is a web development platform that provides the services necessary for developers to build enterprise-class web applications.

\section{- $\quad$ Microsoft SQL Server}

A Relational Database Management System (RDBMS) to store the new user registration and other details in the database.

Flowchart of the Proposed System
International Journal of Computer Applications (0975 - 8887) Volume 175-No. 16, September 2020

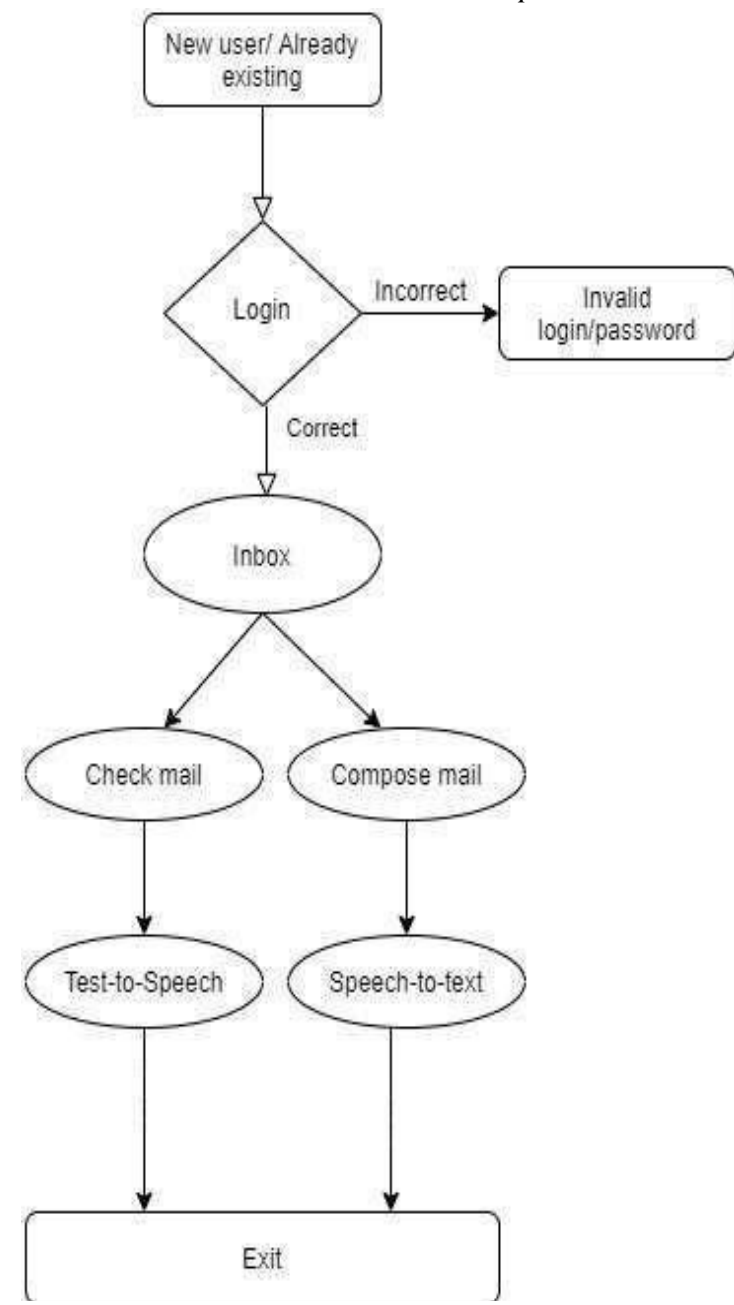

Figure 2: Flowchart of the Proposed System

Fig. 2 explains the flow of the application:

- The user has to register themselves by creating their account.

- It will prompt for Email id and Password, following which, the authentication will take place.

- For correct credentials entered the user's registration process will be successfully completed.

- The user can log into their mail systems, Check

- Mails and compose them.

- Here, the Speech-to-text \& Text-to-speech modules

- Come handy.

- System reads out mails, the sender's name, the subject and the main body.

- $\quad$ Similarly, users can compose emails with the help of the Speech Recognition module or directly send audio attachments with the recorder provided by the application.

The next section discusses the implementation details.

\section{IMPLEMENTATION DETAILS}

This section presents the details of implementation based on the proposed system.

In reference to fig. 3, the new user registration page is used for storing email in the database. (List every entry needed for 
registration) The credentials required for the registration process are the Email ID and Password.

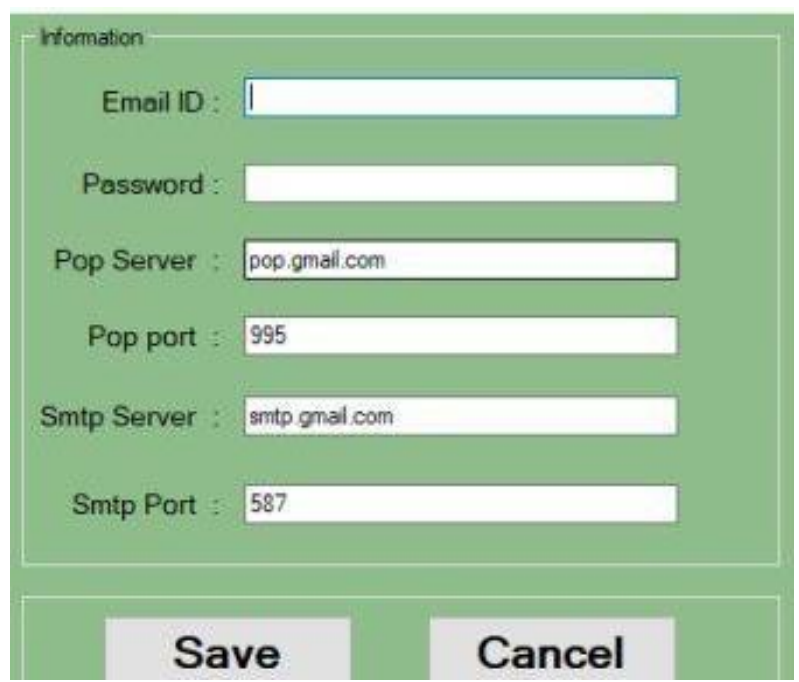

Figure 3: New User Registration Page

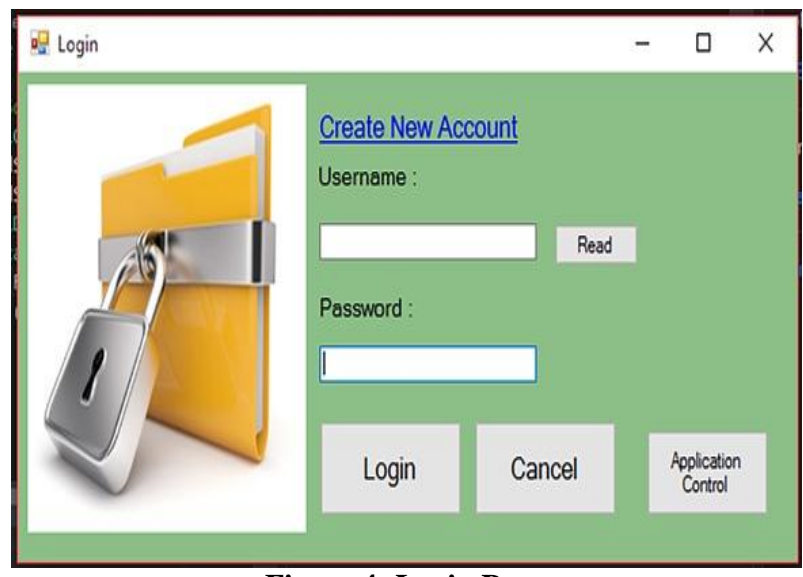

Figure 4: Login Page

In reference to fig. 4 , users have to enter login credentials.

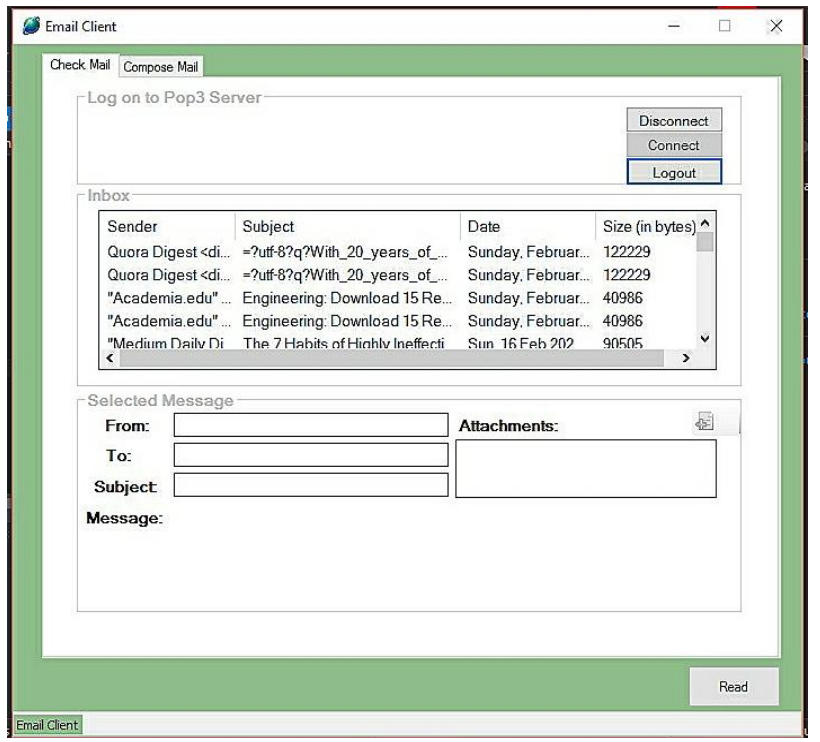

Figure 5: Inbox Page

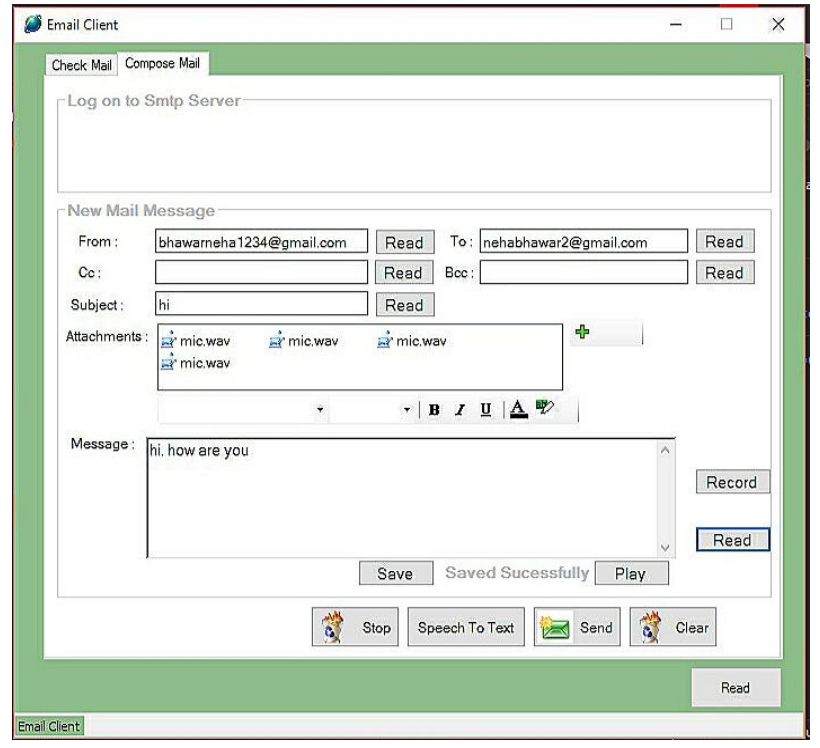

Figure 6: Compose Mail Page

In reference to fig. 5, reading of messages is done perfectly with $100 \%$ accuracy. This is done with the help of a Text-tospeech converter.

In reference to fig. 6 for composing of Emails, we have given a recorder to record messages. The accuracy of speech-to-text is low as there is a need to train it. With the help of the recorder, users can directly attach the audio files.

Thus, we have created a desktop application that makes checking mails and composing mails easier for the visually impaired with the help of Speech-to-text and Text-to-speech converters.

We overcame the limitation of creating a user based mailing system by building the application using Gmail's SMTP and POP3 clients.

This will not only ensure user's data security but also give users a sense of secure mailing.

\section{CONCLUSIONS}

This paper is the proposed Voice based Email system for visually impaired people, which is developed as an application which helps the blind and handicapped people to access mails easily and efficiently. It provides a voice based mailing service where the visually impaired person could read and send mail by their own without the help of others. It requires basic information about keyboard shortcuts. System has eliminated all these concepts and overcome all difficulties faced by the visually impaired. It uses a speech recognition application which provides an efficient voice input method for mailing devices for blind. It is also useful for handicapped and illiterate people.

In future, we attempt to make the system keyboard free and fully voice based. So it's easy for the visually impaired people to access the services. The system developed now is working only on desktops. As use of mobile phones is emerging as a trend today, there is a scope to incorporate this facility as an application in mobile phones also. Also, security measures to be implemented during the login phase can be revised to form the system safer.

\section{REFERENCES}

[1] Sharma, J. and Sharma, J., 2016. Voice Based Mail System. [online] Scribd. Available at: <https://www.scribd.com/document/306826969/voice- 
based-mail-system> [Accessed 29 June 2020].

[2] Amritha Suresh, Binny Paulose, Reshma Jagan and Joby George, "Voice Based Email for Blind". International Journal of Scientific Research in Science, Engineering and Technology (IJSRSET ) - Volume 2, Issue 3, 2016, pp. 93-97.

[3] Milan Badigar, Nikita Dias, Jemima Dias and Mario Pinto, "Voice Based Email Application For Visually Impaired. International Journal of Science Technology \& Engineering (IJSTE) - Volume 4, Issue 12, June 2018, pp. 166-170.

[4] Pranjal Ingle, Harshada Kanade and Arti Lanke, "Voice Based email System for Blinds". International Journal of Research Studies in Computer Science and Engineering (IJRSCSE)- Volume 3, Issue 1, 2016, pp. 25-30.

[5] Bishal Kalita and Santosh Kumar Mahto, "Voice Based Email for Blind People". International Journal of Engineering Science and Computing (IJESC) - Volume 9, Issue 10, October-2019, pp. 23789-23799.

[6] Saurabh Sawant, Amankumar Wani, Sangharsh Sagar, Rucha Vanjari and M R Dhage, "Speech Based E-mail System for Blind and Illiterate People". International
Research Journal of Engineering and Technology (IRJET) - Volume 05, Issue 04, April-2018, pp. 2398-2400.

[7] Dudhbale, P., Wankhede, J.S., Ghyar, C.J., and Narawade, P.S., "Voice Based System in Desktop and Mobile Devices for Blind People". International Journal of Scientific Research in Science and Technology, 4, 2018, pp. 188-193.

[8] Akif Khan, Shah Khusro, Badam Niazi, Jamil Ahmad, Iftikhar Alam and Inayat Khan, "Tetra Mail: A usable email client for blind people". Universal Access in the Information Society-04 September 2018.

[9] Jagtap Nilesh, Pavan Alai, Chavhan Swapnil, Bendre M.R.," Voice Based System in Desktop and Mobile Devices for Blind People". International Journal of Engineering Technology and Advanced Engineering (IJETAE) - Volume 4, Issue 2, February-2014, pp. 404407.

[10] Prof. Umesh A. Patil, Pranouti B. Patil, Teja P. Magdum, Shweta K. Goud and Latika R. Bhosale, "A Survey on Voice Based Mail System for Physically Impaired Peoples". International Journal of Innovative Research in Computer and Communication Engineering (IJIRCCE) Volume 4, Issue 1, January 2016, pp. 1002-1006. 